\title{
Atmospheric phase correction using the CARMA paired antennas calibration system
}

\author{
Laura M. Pérez ${ }^{a}$, James W. Lamb ${ }^{b}$, David P. Woody ${ }^{b}$, B. Ashley Zauderer ${ }^{c}$, John M. \\ Carpenter $^{a}$, Alberto D. Bolatto ${ }^{c}$, Erik M. Leitch ${ }^{b}$, Daniel P. Marrone ${ }^{d}$, Lee G. Mundy, \\ Richard L. Plambeck ${ }^{e}$, Peter J. Teuben ${ }^{c}$, and Melvyn C. H. Wright ${ }^{e}$ \\ ${ }^{a}$ California Institute of Technology, Department of Astronony, 1200 East California Blvd, \\ Pasadena, CA 91125, USA; \\ ${ }^{b}$ Owens Valley Radio Observatory, California Institute of Technology, Big Pine, CA 93513, \\ USA; \\ CUniversity of Maryland, Department of Astronomy, College Park, MD 20742-2421, USA; \\ University of Chicago, Department of Astronomy and Astrophysics, 5640 S. Ellis Ave. \\ Chicago, IL 60637, USA; \\ ${ }^{e}$ University of California at Berkeley, Astronomy Department, 601 Campbell Hall, Berkeley, \\ CA 94720-3411, USA
}

\begin{abstract}
High angular resolution observations are essential to understand a variety of astrophysical phenomena. The resolution of millimeter wave interferometers is limited by large and rapid differential atmospheric delay fluetuations. At the Combined Array for Research in Millimeter-wave Astronomy (CARMA) we have employed a Paired Antenna Calibration System (C-PACS) for atmospheric phase compensation in the extended array configurations (up to $2 \mathrm{~km}$ baselines). We present a description of C-PACS and its application. We also present successful atmospheric delay corrections applied to science observations with dramatic improvements in sensitivity and angular resolution.
\end{abstract}

Keywords: CARMA, C-PACS, atmospheric phase measurement and compensation, millimeter wavelength seeing

\section{INTRODUCTION}

As electromagnetic waves from an astronomical radio source propagate through the atmosphere, sorne emission is lost due to absorption, waves defect due to refraction, and the irregular distribution of water vapor in the atmosphere causes phase fluctuations of these signals, ${ }^{1}$ along the line of sight of each interferometer elenent.

At millimeter and submillimeter wavelengths, the ionosphere has negligible influence, and the primary cause of phase disturbance is the turbulent water vapor distribution in the troposphere, though dry air curbulence may also be important under some circumstances. Atmospheric phase fluctuations reduce the time-averaged amplitude of the signal, and linit the angular resolution of an interferometer (for a review see Ref. 2 and 3 ). As science moves forward, increased angular resolution and higher frequencies are pursued, making it more challenging to achieve a correction for these wavefront distortions. To overcome the effect of atmospheric delay fluctuations several techniques can be employed:

- Self-calibration: in this method, the visibility phase used to calibrate the data is measured from the actual science target. The major advantage of this technique is that the wavefront distortions along the line of sight can be completely removed. The primary disadvantages are that a model for the source spatial structure is required, and this technique can only be applied to bright sources, in order to measure the fringe phase with a high signal to noise ratio in a short integration.

Further author information: (Send correspondence to L.M.P.)

L.M.P.: E-mail: lperezosistro.caltech.edu.

Ground-based and Airborne Telescopes III, edited by Larry M. Stepp, Roberto Gilmozzi, Helen J. Hall Proc. of SPIE Vol. 7733, 77333T · (C) 2010 SPIE · CCC code: 0277-786X/10/\$18 · doi: 10.1117/12.857408 
- Water Vapor Radiometry4,5 (WVR): in this scheme, a dedicated radiometer monitors the water vapor emission along the pointing axis of the antema. As in the self-calibration technique, the major advantage of WVR is that it probes the atmospheric distortions along the line of sight towards the science target. The main drawback is that it requires a physical model of the atmosphere to relate the water line brightness and the path correction, as well as it assumes that refractivity fluctuations are produced by water vapor, which might not always be the case.

- Fast position switching ${ }^{2}, 6$ : in this approach, the interferometer antennas frequently observe a close-by, bright, and point-like calibrator, to measure and correct the rapid phase fuctuations. This method can probe the atmosphere close to the line-of-sight towards the science target, but its main limitation is that it reduces the time spent on the science source by a factor of $\sim 2$.

- Paired antennas ${ }^{7,8}:$ in this scheme, each antema in the interferometer is paired with a monitoring antenna that cont imuously measures the atmospheric delay fluctuations, by observing a nearby, bright, and point-like reference calibrator. Two arrays of closely paired antennas are needed, antennas belonging to the "science array" will observe the science target and phase calbrator, while antemas belonging to the "reference array" simultaneously monitor an atmospheric calibrator. The wavefront correction is accomplished by subtracting the visibility phase measured from the atmospheric calibrator on each baseline of the array. The main advantage of the paired antenna technique is that it accounts for all sources of atmospheric phase fluctuations, either due to water vapor and/or the dry air component, which might not be negligible. ${ }^{9}$ Another advantage is that by having a dedicated array to monitor the atmospheric fuctuations, the time spent observing the science target with the science array is greatly increased. The main disadvantage is that two sets of antemas are required, in pads very close to one another, and is essential that the monitoring array has an adequate phase accuracy and phase stability, in order to apply the correction to the science array without introducing large phase errors.

The Combined Array for Research in Millimeter-wave Astronomy (CARMA) has implemented the CARMA Paired Antenna Calibration System (C-PACS) to provide atmospheric phase measurement and compensation on the longest baselines of the interferometer (up to $2 \mathrm{~km}$ in length). The goal of C-PACS is to enable routine inaging in the most extended CARMA configurations. C-PACS will allow us to reach unprecedented angular resolutions at the 1.3 and 2.7 rmm bands with CARMA, making it possible to resolve the emission from compact sources. In this paper we present a description of CARMA and the C-PACS instrument in section 2, the calibration technique is described in section 3, successful atmospheric delay corrections applied to science observations with their improvements in sensitivity and angular resolution are discussed in section 4 , and final remarks are given in section 5 .

\section{C-PACS DESCRIPTION}

CARMA is a heterogeneous millimeter interferometer comprising 23 antennas: nine $6.1 \mathrm{~m}$ telescopes from the Berkeley-illinois-Maryland Association (BIMA), six $10.4 \mathrm{~m}$ telescopes from the California Institute of Technology/Owens Valley Radio Observatory (OVRO), and eight $3.5 \mathrm{~m}$ telescopes from the University of Chicago Sunyaev-Zel'dovich Array (SZA). The array can be arranged into 5 different configurations, with the most extended ones having baselines that range in length from 100 to $1000 \mathrm{~m}$ (B configuration) and 250 to $1900 \mathrm{~m}$ (A configuration), to achieve an angular resolution of $0.3^{\prime \prime}$ and $0.15^{\prime \prime}$ respectively, at an observing frequency of $230 \mathrm{GHz}$.

During $\mathrm{A}$ and $\mathrm{B}$ configuration season, the eight $3.5 \mathrm{~m}$ antennas are paired with selected $6.1 \mathrm{~m}$ and $10.4 \mathrm{~m}$ antennas, preferably on the longest baselines of the array. The position of the $3.5 \mathrm{~m}$ antemas is typically to the south of the larger antenna, offset by $25 \cdots 30 \mathrm{~m}$. This pad separation is a compromise between the requirement of pairing the antennas as close as possible (to probe the same atmospheric path) and to avoid shadowing and physical collisions between antenna pairs.

The science array of C-PACS, composed of the 6.1/10.4 m antennas, operates in the 1 or 3 mm atmospheric bands as requested by the investigator. The reference array, consisting of the $3.5 \mathrm{~m}$ antennas, operates at $30 \mathrm{GHz}$ 
in C-PACS mode. The atmospheric delay measured at $30 \mathrm{GHz}$ can be used at 100 or $230 \mathrm{GHz}$ because ionospheric scintillation is negligible at these frequencies, ${ }^{10}$ and the refractive index of water vapor at millimeter and centimeter wavelengths has been measured in Ref. 11, and it differs by $<10^{-6}$. The minimal dispersion of the refractive index has been experimentally verified from C-PACS observations (Zauderer et al. in prep). Observations of the same target at millimeter and centimeter wavelengths exhibit phase fuctuations of comparable behavion, that can be scaled from one wavelength to the other by the ratio of the observed frequencies. Therefore, the delay fuctuations inferred from the $30 \mathrm{GHz}$ reference observations are sealed to the frequency of the science array observations by the ratio of their frequencies.

The observing cycle is setup to comprise observations of the science target interleaved with periodic observations of the phase calibrator. During the phase calibrator observations, both science and reference arrays observe the same bright celestial source to determine instrumental phase drifts. Subsequently, the science array observes the science target while the reference array monitors an atmospheric calibrator to measure the delay iritroduced by the atmosphere. In the following section we describe the calibration technique in more detail.

\section{C-PACS CALIBRATION}

The visibility phase $\Phi_{i j}$ measured on baseline $i \cdots j$ of an interferometer can be written as:

$$
\Phi_{i j}(t)=\arg \left(V_{i j}(t)\right)+\phi_{i j}^{i}+2 \pi \nu\left[r_{i j}^{I}(t)+\tau_{i j}^{A}(t)\right]+\epsilon^{R}(t)
$$

where $V_{i j}$ is the true source visibility for this particular baseline, $\nu$ corresponds to the observing frequency, $\tau_{i j}^{I}$ is the instrumental delay, $\tau_{i j}^{A}$ is the atmospheric delay difference between antennas $i$ and $j$ through the line-of-sight, $\phi_{i, j}^{I}$ is the instrumental phase difference between these antennas, and $\epsilon^{R}$ is the contribution from radiometric noise. We have assumed that the baseline vector is precisely known and the geometrical delay can be completely removed. To simplify the following presentation we ignore the radiometric noise. We also assume that any stable bandpass phases introduced by the receiver and the intermediate frequency (IF) system, as well as any phase offset introduced by the local oscillator (LO), can be measured and calibrated with bandpass calibration.

Similar to the discussion in Ref. 7, consider two pairs of antennas: antennas 1 and 2 belong to the science array, while antemas 3 and 4 belong to the reference array. Phase fluctuations are caused by the contribution from the atmospheric delay term, $\tau_{i j}^{A}$, which has no frequency dependence. Hence, Equation 1 can be written in terms of delays for the sets of antenias described here:

$$
\tau_{12}=\Phi_{12} /\left(2 \pi \nu_{12}\right)=\arg \left(V_{12}\right) /\left(2 \pi \nu_{12}\right)+\tau_{12}^{I}+\tau_{12}^{A}
$$

for the seience array, and

$$
\tau_{34}=\Phi_{34} /\left(2 \pi \nu_{34}\right)=\arg \left(V_{34}\right) /\left(2 \pi \nu_{34}\right)+\tau_{34}^{I}+\tau_{34}^{A}
$$

for the reference array. The temporal dependence from these equations has been renoved for clarity. The frequency of observation for the science array and reference array are denoted by $\nu_{12}$ and $\nu_{34}$ respectively. The reference array monitors a point source at the interferometer phase center, and hence $\arg \left(V_{34}\right)=0$.

In the paired antema system the baseline pairs from each array will produce near-simultancous visibility phase measurements. To apply the C-PACS correction, the delay measured on the reference baseline $\left(\tau_{34}\right)$ is subtracted from the science baseline delay $\left(\tau_{12}\right)$ on every integration. Applying the correction to the science target (ST) data and the phase calibrator (PC) data yields

$$
\begin{aligned}
\left(\Delta \tau_{12}\right)_{\mathrm{ST}} & =\left(\tau_{12}-\tau_{34}\right)_{\mathrm{ST}} \\
& =\arg \left(V_{12}\right) /\left(2 \pi \nu_{12}\right)+\left[\tau_{12}^{I}-\tau_{34}^{I}\right]+\left[\tau_{12}^{A}-\tau_{34}^{A}\right] \\
& -\cdots \arg \left(V_{12}\right) /\left(2 \pi \nu_{12}\right)+\left[\tau_{12}^{I}-\tau_{34}^{I}\right]
\end{aligned}
$$

and

$$
\begin{aligned}
\left(\Delta \tau_{34}\right)_{\mathrm{PC}} & =\left(\tau_{12}-\tau_{34}\right)_{\mathrm{PC}} \\
& =\left[\tau_{12}^{I}-\tau_{34}^{I}\right]+\left[\tau_{12}^{A}-\tau_{34}^{A}\right] \\
& =\left[\tau_{12}^{I}-\tau_{34}^{I}\right]
\end{aligned}
$$


The atmospheric delay difference between the science array and the reference array cancel $\left(\tau_{12}^{A}-\tau_{34}^{A}=0\right)$, if the atmospheric calibrator and science target are observed simultaneously and their separation in the sky is small. The slow varying instrumental difference delay that remains $\left(\tau_{12}^{\prime} \cdots \tau_{34}^{\prime}\right)$ can be removed by phase referencing to the phase calibrator. Therefore the calibrated visibility phase difference on the science target becomes:

$$
\Delta \Phi_{12}=2 \pi \nu_{12}\left(\left(\Delta \tau_{12}\right)_{\mathrm{ST}}-\left(\Delta \tau_{34}\right)_{\mathrm{PC}}\right)=\arg \left(V_{12}\right)
$$

which corresponds to the source fringe phase between antemnas 1 and 2 , the quantity we are interested in recovering.

We now describe how the atmospheric delay is measured and applied to correct the science data. In the C-PACS tcehnique this correction is performed in two steps: we neasure a delay from the cm-wave observations of the atmospheric calibrator, and then this atmospheric delay is applied to each corresponding paired baseline in the science array. Only eight of the 15 antennas from the science array are paired with a reference antema, and only those 8 science antennas will have the C-PACS correction applied (this generally correspond to the antemas that suffer the most decorrelation, since they are located in the outskirts of the array). The delay derived from the reference array is applied to the science target and phase calibrator on each record (a record is one integration of the interferometer, as setup by the investigator, and it can range from a fraction of a second to several seconds). The delays are computed from the mean phase across all channels in the $30 \mathrm{GHz}$ observations divided by the mean frequency.

After applying the delay corrections at each integration record, a long-interval (generally 10-15 minutes long) phase gain calibration is applied to the $\mathrm{mm}$-wave data to remove the slowly varying instrumental delay difference from the two different arrays, and with this we recover equation (6) on each baseline of the array.

\section{APPLYING C-PACS TO SCIENCE OBSERVATIONS}

In the last two northern winter seasons (November 2008 to February 2009, and November 2009 to February 2010) the C-PACS system has been put into practice at CARMA. An example of a successful atmospheric phase correction is presented in this section.

The C-PACS calibration technique was applied to observations of the circumstellar dust around PP 13S*, a young star located in the constellation of Perseus and embedded in the L1473 dark clond at a distance of $\sim 350$ parsec. ${ }^{12}$ The star is surrounded by an extended disk/envelope that contains about 0.6 solar masses of gas and dust. ${ }^{13}$ These new observations using C-PACS rneasure the distribution of dust continum emission on spatial scales as small as $\sim 0.15^{\prime \prime}$.

Figure 1 shows the resulting maps, obtained employing natural weighting, before and after applying the C-PACS correction to the data. No absolute flux scale or amplitude gain calibration have been applied to this data to only consider the effect of implementing C.PACS. This data was obtained in two separate days, and figures (1a) and (1b) show the resulting images obtained on each day. When all the data are combined into a single map, the effect of applying the C-PACS correction becomes evident, as shown in figures (1c) without C-PACS and (1d) with C-PACS. The synthesized beam of the final map corresponds to $0.15^{\prime \prime} \times 0.14^{\prime \prime}$. The color scale range is the same for all maps, and all contours start at $5 \sigma$, where $\sigma$ is the noise level in the respective map indicated on the figure.

C-PACS improved each of the observations obtained in A and B configurations, despite the different weather conditions and baseline lengths of these observations. The improvements on the image quality can be quantified from three image properties: the peak of the surface brightness distribution on the map, the noise (or RMS) level of the map, and the observed source size. The peak flux density measured on each map increased when C-PACS was applied; in the combined $A+B$ map, the peak fux increased from $42.2 \mathrm{mJy}$ to $67.8 \mathrm{mJy}$ ( $\mathrm{a}$ factor of 1.6 increase) when applying the C-PACS correction. The noise level for all maps diminished when C-PACS was applied; in the combined A+B map, the noise decreased from $\sigma=1.54 \mathrm{mJy} /$ beam (Figure (1c)) to $\sigma=-1.14 \mathrm{mJy} /$ beam (Figure (1d)), which corresponds to a $35 \%$ improvement. Similarly, the surface brightness distribution changes after applying C-PACS, with the peak flux density increasing and the source becoming sharper, with its size diminishing from $0.41^{\prime \prime} \times 0.27^{\prime \prime}$ to $0.27^{\prime \prime} \times 0.26^{\prime \prime}$ (a $26 \%$ decrease in the source full width at half maximum or FWHM). 

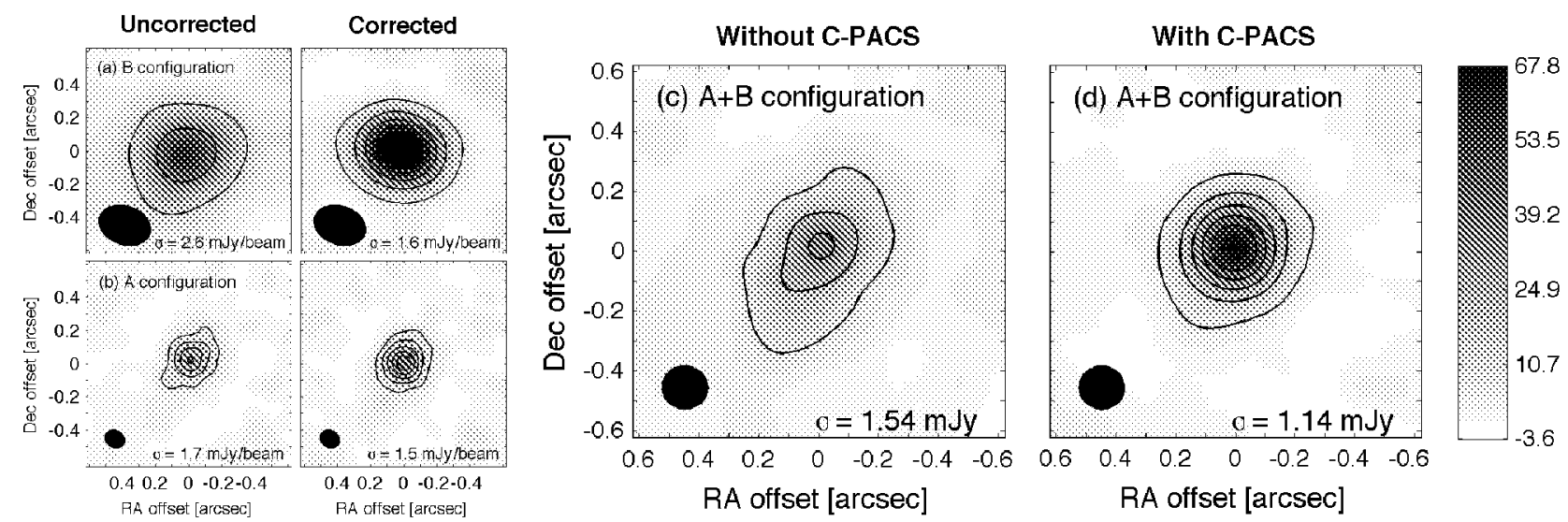

Figure 1. Figures (1a) and (1b) show the PP 13S* continum maps obtrined on 2 separate days in $A$ and $B$ configurations, with (right column) and without (Ieft column) C-PACS correction. Figures (1c) and (1d) show the combined AtB configuration continum map of PP 135*, whithout (middle panel) and with (left panel) C-PACS correction. The color scale range is same for all maps (from -3.6 to $67.8 \mathrm{~m}$ Jy) such that the measured fiuxes can be compared directly. Solid contours start at $5 \sigma$, where $\sigma$ is the noise level in the corresponding map. For (1a), (1c) and (1d) maps, solid contours increase by steps of $10 \sigma$. For (1b) the solid contouts increase by steps of $5 \sigma$. (Adapted from Pérez et al. in prep.)

\section{FINAL REMARKS}

The angular resolution of millimeter wave interferometers is limited by the delay fuctuations introduced by the atmosphere along the line of sight of each interferometer element. At CARMA we have employed a paired antennas approach to calibrate the atmospheric wavefront distortions. We have demonstrated that C-PACS can calibrate atmospheric phase fuctuations on the longest baselines of the array during regular science observations. Several improvements on the data quality were noticed when the C-PACS correction was applied, in particular for the case of PP 135* an increase in the peak flux by a factor of 1.6 , a decrease on the image noise level by $35 \%$, and a $26 \%$ decrease in the observed size of this object. The radiometric noise introduced from the reference array to the science array, as well as the projected beam separation of antenna pairs (at the height of the turbulent layer), are the main limitations for the improvement in the phase error and amplitude coherence warranted by the paired antennas technique. Future improvements being considered at CARMA will be along the lines of increasing the sensitivity of the reference array, to be able to take advantage of fainter calibrators, as well as correcting the non-paired baselines by using a weighted scheme of correlations between the paired antennas.

\section{ACKNOWLEDGMENTS}

Support for CARMA construction was derived from the Gordon and Betty Moore Foundation, the Kenneth T. and Eileen L. Nom is Foundation, the James S. McDonnell Foundation, the Associates of the Califomia Institute of Technology, the University of Chicago, the states of California, Mlinois, and Maryland, and the National Science Foundation. Ongoing CARMA development and operations are supported by the National Science Foundation under a cooperative agreement, and by the CARMA partner universities. LMP acknowledges support for graduate studies through a Fulbright-CONICYT scholarship.

\section{REFERENCES}

[1] Thompson, A. R., Moran, J. M., and Swenson, Jr., G. W., [nterferometry and Synthesis in Radio Astronomy, 2nd Edition] (Apr. 2001).

[2] Lay, O. P., "Phase calibration and water vapor radiometry for millimeter-wave arrays," A6 6 AS 122, 547-557 (May 1997).

[3] Lay, O. P., "The temporal power spectrum of atmospheric fluctuations due to water vapor," A 65 , 122 , $535-545$ (May 1997). 
[4] Westwater, E. R., "An analysis of the correction of ray errors due to atmospheric refraction by microwave radiometric techniques;" ESSA Technical Report IER 30-ITSA 30 (1967).

[5] Woody, D., Carpenter, J., and Scoville, N., "Phase Correction at OVRO Using $22 \mathrm{GHz}$ Water Line Monitors," in [Imaging at Radio throngh Submillimeter Wavelengths], J. G. Mangum \& S. J. E. Radford, ed., Astronomical Society of the Pacific Conference Series 217, 317- (2000).

[6] Holdaway, M., Radford, S. J. E., Owen, F. N., and Foster, S. M., "Fast Switching Phase Calibration: Effectiveness at Mauna Kea and Chajnantor," MMA Memo NRAO 139 (1995),

[7] Asaki, Y., Saito, M., Kawabe, R., Morita, K., and Sasao, T., "Phase compensation experiments with the paired antermas method," Radio Science 31, 1615-1626 (1996).

[8] Asaki, Y., Shibata, K. M., Kawabe, R., Roh, D., Saito, M., Morita, K., and Sasao, T., "Phase compensation experiments with the paired antemas mothod 2. Milineter-wave fringe correction using centimeter-wave reference," Rodio Science 33, 1297-1318 (1998).

[9] Stirling, A, Richer, J, Hills, R, and Pardo, J., "Phase correction for ALMA," in [Society of Photo-Optical Instrumentation Engineers (SPIE) Conference Series], Society of Photo-Optical Instrumentation Engineers (SPIE) Conference Series 6267 (July 2006).

[10] Hales, S., Hills, R., Robson, Y., Richer, J., Delgado, G., Otrola, A., and Radford, S., "Investigation of Anomalous Fast Phase Fluctuations in the Site-Test Interferometer Data from Chajnantor," AlMA Memo NRAO 459 (2003).

[11] Hill, R. J., "Dispersion by atmospheric water vapor at frequencies less than $1 \mathrm{THz}, "$ Antennas and Propagation, IEEE Transactions on 36, 423-430 (1988).

[12] Cohen, M., Aitken, D. K., Roche, P. F., and Williams, P. M., "The unique cometary nebula Parsamian 13," ApJ 273, 624-632 (Oct. 1983).

[13] Sandell, G. and Aspin, C., "PP 13S, a young, low-mass FU Orionis-type pre-main sequence star," A 83 A 333, 1016-1024 (May 1998). 